\title{
The Search for Bacteria and Viruses in the Stratosphere
}

\author{
N. Chandra Wickramasinghe ${ }^{1,2,3,4}$, Dhammika Maganarachchi ${ }^{4}$, Robert Temple ${ }^{5}$, Daryl H. Wallis ${ }^{3}$, \\ Edward J. Steele ${ }^{2,6}$, Meththika Vithanage ${ }^{7}$, Reginald M. Gorczynski ${ }^{8}$, Gensuke Tokoro ${ }^{4}$, Brig Klyce ${ }^{9}$ \\ and Predrag Slijepcevic ${ }^{10}$ \\ ${ }^{1}$ Buckingham Centre for Astrobiology, University of Buckingham, UK \\ ${ }^{2}$ Centre for Astrobiology, University of Ruhuna, Matara, Sri Lanka \\ ${ }^{3}$ National Institute of Fundamental Studies, Kandy, Sri Lanka \\ ${ }^{4}$ Institute for the Study of Panspermia and Astroeconomics, Gifu, Japan \\ ${ }^{5}$ History of Chinese Science and Culture Foundation Conway Hall, London, UK \\ ${ }^{6}$ C. Y. O'Connor, ERADE Village, Foundation, Piara Waters, Perth 6112 WA, Australia \\ ${ }^{7}$ University of Sri Jayawardenepura, Kelaniya, Sri Lanka \\ ${ }^{8}$ University Toronto Health Network, Toronto General Hospital, University of Toronto, Canada \\ ${ }^{9}$ Astrobiology Research Trust, Memphis, TN, USA \\ ${ }^{10}$ Brunel University, London, UK \\ Email: ncwick@gmail.com
}

\begin{abstract}
As we enter a new decade a plethora of evidence pointing to our cosmic origins is close to the point of being overwhelming. The possibility that viruses and bacteria of extraterrestrial origin are implicated in the onset and propagation of pandemic diseases, although still a controversial hypothesis, is also coming to the fore as one of the more plausible explanations of recent epidemiologic data. A long-entrenched paradigm of Earth-centred biology appears to prevail in scientific culture, which we believe is stifling serious concern for monitoring the Earth's stratosphere for incoming bacteria and viruses and in consequence our safety. A matter of crucial importance for the next decade is to carry out a single decisive experiment that is long overdue - establishing empirically that extraterrestrial microbiota are continually being resupplied to the stratosphere and reaching Earth in biologically significant numbers.
\end{abstract}

Keywords: stratospheric dust, comets, bacteria and viruses, panspermia

\section{Introduction}

Four decades ago Fred Hoyle and one of the present authors had accumulated enough supportive evidence to be confident in our assertion that terrestrial life and terrestrial biology were inextricably linked to the cosmos at large. The main connecting link was comets and cometary debris that continuously gain entry to the Earth's environment. Supportive evidence for this position was multi-disciplinary with the result that a majority of scientists working within the confines of their own speciality remained loathe to transgress the limitation of their particular boundaries and to contemplate a wider cosmic world view.

In a public lecture by Fred Hoyle delivered in Cardiff in 1980 he expressed our views as follows:

"The very small probabilities, which one calculated for the assembly of these substances (eg enzymes), demonstrates as near to certainty as one would wish that life did not originate here on the Earth. Indeed, the infinitesimal probabilities demonstrate that life is even too complex for its origin to be confined within our galaxy alone. The resources of the whole universe were almost certainly needed......"

Thus were sown the first seeds of the theory of life as a cosmic phenomenon that were developed in subsequent decades. From the very earliest days with the development of new areas of science and new technologies the predictions that followed from this single unifying hypothesis of cosmic life were amenable to examination, and they survived the well-known Popperian test with unfailing accuracy (Steele et al, 2018, 2019). 
In the discussion that follows we have confined our attention to one particular test - perhaps the most obvious smoking gun for the new paradigm which had to be established and conceded as an irrefutable fact. The emergence of life on Earth and its further evolution is in our view contingent on the continuing arrival of biological material (in the form of genes) from the wider cosmos. The adaptation and hybridisation of extraterrestrial genes within evolving lines, if it is firmly established, has profound implications. Thus we predict that confirming or refuting the possibility of the ongoing entry of novel genes - from extraterrestrial bacteria and viruses - is a matter of paramount importance for a correct understanding of biology, even perhaps for our very survival.

\section{Early Evidence}

An obvious place to discover biological material from comets is in the Earth's upper atmosphere. Cometary meteoroids (fragments of comets) and interplanetary dust particles are known to enter the atmosphere in vast quantity, at a more or less steady rate, averaging $\sim 10^{2}$ tonne/day. Although much of this incoming material burns up as meteors, a significant fraction survives entry and can reach the ground. Organic grains, including bacteria and viruses of micrometre sizes, arriving as clumps and dispersing in the high stratosphere, would be slowed down gently and would not be destructively heated (Hoyle and Wickramasinghe, 1979). The Earth's atmosphere could thus serve as an ideal collector of biological material which can provide the potential for both random pandemics and further evolutionary progress.

The Australian scientist E.K. Bigg (1) was among the first to actually recover stratospheric particles from above 25km using balloons. His collections dating from 1960 onward clearly reveal the presence of oval shaped dust particles, and particles endowed with fimbria, which Bigg himself speculated may have a possible biological provenance. A few years later Greene et al (2) carried out a series of balloon flights to recover microorganisms in the two height ranges 30,000-60,000 ft and 60,000 - 90,000 ft in the stratosphere. Positive results in both these height ranges possibly pointed to an infalling microbial population, and this came as an unwelcome surprise in the years running up to the dawn of the space age. Although some of the microorganisms collected in early experiments were claimed to exhibit "unusual" properties, such as pigmentation and radiation resistance, their possible extraterrestrial origin remained a matter of dispute. No DNA sequencing procedure was available at the time to ascertain significant deviations there might have been from any related terrestrial species. Furthermore, the collection and laboratory techniques available at the time left open a high chance of contamination. A history of early attempts to probe the stratosphere for microorganisms is summarised by Smith (2013).

Methods for the collection of cometary microbiota in the stratosphere, if they are to be decisive, should involve procedures for either sifting out a terrestrial component or for excluding such contamination altogether. In 2001 the Indian Space Research Organisation (ISRO) launched a balloon into the stratosphere to heights between 20 and $41 \mathrm{~km}$ to collect stratospheric air under aseptic conditions with a view to testing the theory of cometary panspermia (Harris et al., 2002). The procedure involved the use of cryogenically cooled, stainless steel cylinders that were evacuated and autoclaved and fitted with valves that could be opened at predetermined altitudes. A large quantity of stratospheric air at $41 \mathrm{~km}$ was collected in this manner and the cylinders containing this material parachuted back for laboratory study.

The air within the pressurised cylinders was carefully released and passed through millipore membrane filters under carefully controlled laboratory conditions. Aerosols (dust and bacteria) present within the volume of stratospheric air were thus trapped and collected on the filters. The particles recovered fell into two broad categories: (a) mineral grain aggregates, very similar to Brownlee particles (inorganic interplanetary dust), and (b) fluffy carbonaceous aggregates which could be identified as clumps of bacteria (see Fig. 1a). Typical dimensions of the clumps were about $10 \mu \mathrm{m}$.

In addition to structures such as shown in Fig. 1(a), which were presumed to be bacteria, the stratospheric samples from 2001 also revealed evidence of similar-sized bacterial clumps that could not be cultured but were detected by the use of a fluorescent dye (carbocyanine dye). The uptake of the dye revealed the presence of living cells in the clumps (see Fig.1b) which were interpreted to be "viable but non-culturable microorganisms." The cometary origin of all such particles was inferred on the grounds that the altitude of $41 \mathrm{~km}$ was too high for lofting 10-um sized clumps of solid material from the Earth's surface under normal conditions. 


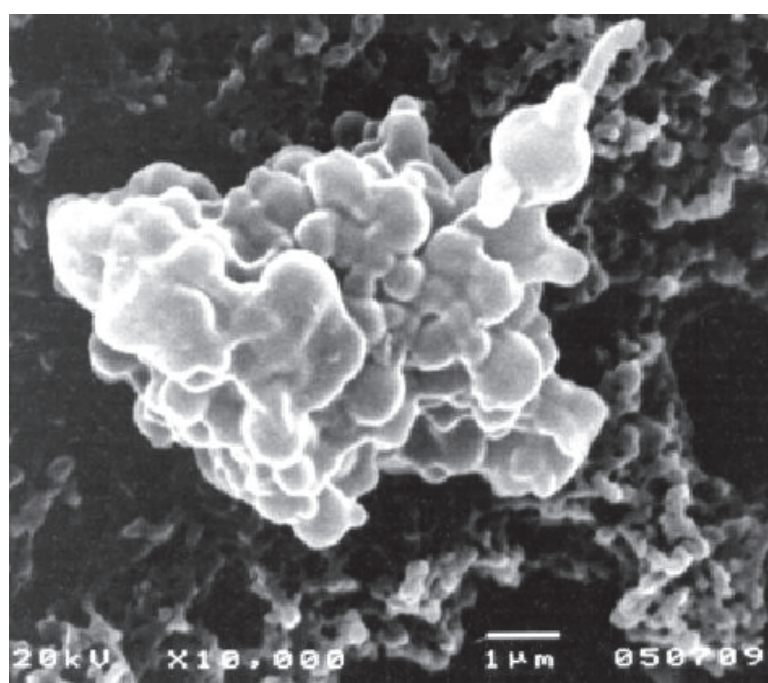

(a)

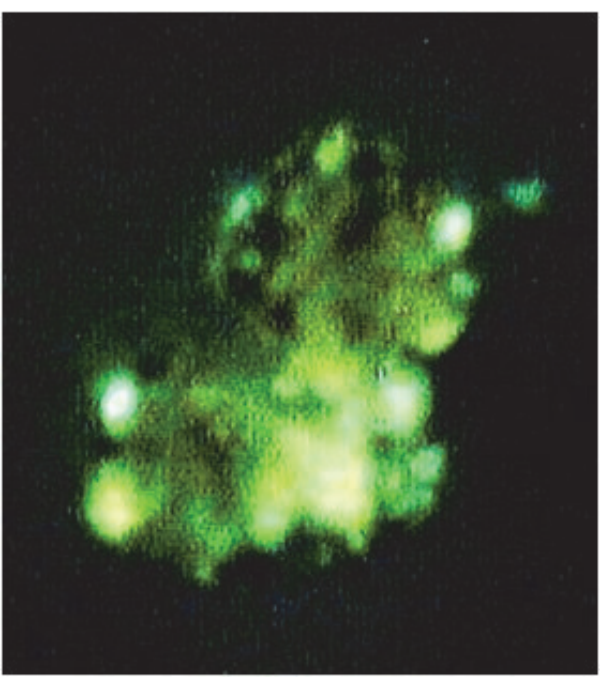

(b)

Figure 1. a) A carbonaceous stratospheric particle from $41 \mathrm{~km}$ resembling a clump of cocci and a rod bacterium. b) Clump of viable bacteria which could not be cultured further.

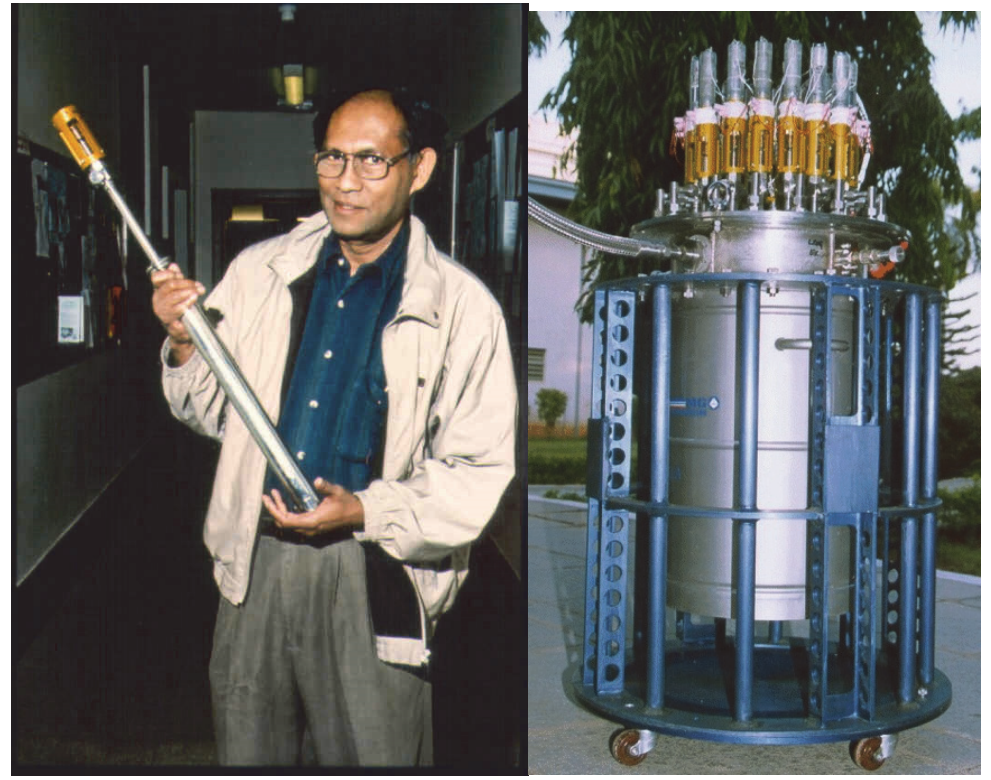

Figure 2. L: Cylinder containing stratospheric air; R: stack of cylinders each with 1.3 litre volume and launched within a cryogenic liquid neon container. Each evacuated cylinder fills with air at $41 \mathrm{~km}$ and when parachuted back to Earth contains air and aerosols at high pressures that need to be released slowly and with caution.

\section{Flux of Microbes from Space}

It was next possible to infer the in-fall rate of biological particles at $41 \mathrm{~km}$ from the data obtained in the balloon launch of 2001 (Harris et al, 2002). When the high pressure stratospheric air within the recovered cylinders was passed through millipore filters Harris et al (2002) discovered an average of one clump of putative biological cells per $25 \mathrm{~mm}^{2}$ of filter area. With a total membrane filter area of $\sim 2000 \mathrm{~mm}^{2}$ and given the total NTP volume of air released, the entire membrane filter would thus have have contained 80 clumps of cells representing the bacterial component of the air within the cylinder that was collected 
from $\sim 41 \mathrm{~km}$. With the volume of air released having been recorded as 18.5 litre the NTP concentration of clumps is thus $\sim 80 / 18.5=4.3$ per litre. Converting this to the ambient conditions at the point of collection $\sim 39-41 \mathrm{~km}\left(\mathrm{P}=2.9 \times 10^{-3}\right.$ bar, $\left.\mathrm{T}=253 \mathrm{~K}\right)$ we obtain a local clump density of $1.4 \times 10^{-2}$ per litre of ambient stratospheric air at $40 \mathrm{~km}$ altitude.

Next, we need to estimate the falling (terminal) speed of aerosols falling through the stratosphere at various heights. For this we use data set out by Kasten (1964) confirmed by later studies of Colbeck (2001). From Kasten's figures we can calculate the falling speeds of spherical particles of various radii and unit specific gravity as shown in Table 1:

Table 1. Falling speed w $(\mathrm{cm} / \mathrm{s})$

\begin{tabular}{|c|c|c|c|c|}
\hline${ }_{\mathrm{z}(\mathrm{km})} a($ micron $)$ & 0.3 & 1 & 3 & 10 \\
\hline 40 & 0.12 & 0.36 & 1.52 & 5.37 \\
\hline 20 & 0.0066 & 0.036 & 0.187 & 1.69 \\
\hline 10 & 0.0019 & 0.017 & 0.137 & 1.52 \\
\hline
\end{tabular}

The average mass for a 3 micron radius clump can be taken as $\sim 3 \times 10^{-11} \mathrm{~g}$, and the settling speed of such a clump at $40 \mathrm{~km}$ according to Table 1 is $\sim 1.52 \mathrm{~cm} / \mathrm{s}$. For the entire Earth's surface of $5 \times 10^{18} \mathrm{~cm}^{2}$, we thus calculate an infall rate as:

$$
\sim 1.4 \times 10^{-5} \times 3 \times 10^{-11} \times 5 \times 10^{18} \times 1.52 \cong 3.19 \times 10 \mathrm{~g} \mathrm{~s}^{-1} \cong 3 \text { tonnes per day }
$$

This converts to some $10^{8}$ bacteria per square metre arriving from space at the Earth's surface every day, along with possibly ten times as many associated viruses.

\section{Further Stratospheric Sampling}

From 2013 onward Milton Wainwright and his team conducted a series of balloon flights in Chester, England to collect microbes from heights in the range $21-25 \mathrm{~km}$ in the stratosphere (Wainwright et al, 2015). The sampler included a drawer mechanism that could be opened and closed at a predetermined height with new electron microscope stubs in this study then exposed to falling microbes. The particles fell at speeds high enough to crater some of the carbon stubs, and many particles were discovered to be embedded in the stubs when they were recovered. The stubs were examined in the laboratory using techniques of electron microscopy. With controls taken to avoid contamination at every stage, Wainwright et al (2015) discovered 10micron organic structures to which they ascribed a biological provenance. A new group (Magannarachchi et al, 2020) has in planning a proposal to launch a balloon to $50 \mathrm{~km}$ over central Sri Lanka for collection of stratospheric aerosols. The collected material would be examined using a variety of methods including DNA/RNA analysis, and the possible non-terrestrial component of microbial ingress will be identified in this way. In view of the ongoing COVID-19 pandemic, the deepest sunspot minimum in a hundred years and the remarkable increase in the numbers and latitude range of NLC (noctilucent cloud) sightings this project is extremely timely and should be accorded the highest priority (https://spaceweatherarelive.com/2020/03/26/noctilucent-clouds-over-the-south-pacific/).

\section{Distinguishing Terrestrial Microbiota from Space-Incident Bacteria}

The argument has been made that many of the pathogens detected in the studies discussed above as not derived from extraterrestial sources, but are contaminants "uploaded" from earth. Thus the predicted numbers of "in-falling" bacteria estimated in Section 3 should be compared with bacteria (and viruses) originating from the Earth's surface, some of which could conceivably be lofted to heights of about $3 \mathrm{~km}$ in upward air currents and brought down in mist and rain.

Recently Reche et al (2018) measured the total flux of bacteria and viruses reaching near ground level in high mountains and found recycled viruses arrive at the rate of 800 million per square metre per day. Sorting out the component of space incident microbes from ground-level indigenous microbes (bacteria 
and viruses) may not be easy, but would be profoundly important to say the least. With sufficient commitment, the resolution of this problem lies well within the scope currently available technologies.

First and foremost, it will be important to repeat these early attempts to isolate microorganisms from the stratosphere and possibly beyond. In this context it is worth noting that Grebennikova et al (2018) have already isolated microorganisms above the stratosphere on the windows of the International Space Station at 400km and Wickramasinghe and Rycroft (2018) have discussed the difficulties of lofting such particles to reach these heights.

Microbial entities collected in the stratosphere (or above) can be analysed in many ways to establish their space origin. The use of nanosims technology to determine isotopic signatures would be one route of investigation. This analysis was attempted in the case of the 2001 recoveries without success, but these studies should be repeated and extended with contemporary technology. The fact that appropriate facilities are few and far between on the planet, and access is expensive and difficult, remains a limitation that should be overcome.

If both the space incident microorganisms and terrestrial microbes originate from disconnected pieces of a single cosmic biosphere (Earth and space) their genetic differences may well turn out to be subtle and even difficult to detect. Indeed, an ISRO sponsored balloon flight into the stratosphere in 2006 recovered three new bacterial species that are genetically similar ( $80 \%$ homologous) to known terrestrial species but sufficiently different to be classified as different species (Shivaji et al, 2007). The first of the new species recovered from $41 \mathrm{~km}$ was named Janibacter hoylei, after Fred Hoyle. Data such as these should provide further incentive for a more detailed and systematic analysis of the problem. In terms of our species' future ability to act pro-actively, rather than reactively, to novel, potentially pandemic-inducing microorganisms, as illustrated by the current COVID19 pandemic, this issue we would argue is of paramount importance.

\section{References}

1. Allen, C.W.: 1967, Astrophysical Quantities, London Athlone Press.

2. Bigg, E.K.,1983. Particles in the upper atmosphere, in Fundamental Studies and the Future of Science, ed C. Wickramasinghe, University College, Cardiff Press, pp. 38-51.

3. Burch, C.W.: 1967, Symp. Soc. Gen. Microbiol. 17, 345-373.

4. Colbeck, I., (1998) in Physical and Chemical Properties of Aerosols (ed Colbeck, I.) Blackie, Lond

5. Grebennikova, T.V., Syroeshkin, A.V., Shubralova, E.V. et al, 2018. The DNA of Bacteria of the World Ocean and the Earth in Cosmic Dust at the International Space Station, Scientific World Journal 2018: 7360147. Published online 2018 Apr 18. doi: 10.1155/2018/7360147; (https://www.hindawi.com/journals/tswj/aip/ $7360147 /)$

6. Greene, V., Hagberg, C., Lundgren, D., and Pederson, P. (1965) Microbiological exploration of stratosphere: results of six ex- perimental flights. In Proceedings of the Atmospheric Biology Conference, University of Minnesota, 1315 April 1964, NASA, Washington, DC, pp 199-212.

7. Harris, M.J., Wickramasinghe, N.C., Lloyd, D., Narlikar, J.V., Rajaratnam, P., Turner, M.P., Al-Mufti, S., Wallis, M.K. \& Hoyle, F. (2001) The detection of living cells in stratospheric samples. Proc SPIE 4495, 192-198.

8. Kasten, F.: 1968, Falling Speed of Aerosols, J. Appl. Meteorol. 7, 944-947.

9. Reche, I., D'Oria, G, Mlandenov, N. et al, 2018. Deposition rates of viruses and bacteria above the atmospheric boundary layer. The ISME Journal https://doi.org/10.1038/s41396-017-0042-4

10. Shivaji, S., Chaturvedi, P., Begum, Z. et at, 2009. Janibacter hoylei sp. nov., Bacillus isronensis sp. nov. and Bacillus aryabhattai sp. nov., isolated from cryotubes used for collecting air from the upper atmosphere. Int. $J$ Systematics Evol. Microbiol, 59, 2977-2986

11. Smith, D.J., (2013) Microbes in the Upper Atmosphere and Unique Opportunities for Astrobiology Research, Astrobiology, 13(20), DOI: 10.1089/ast.2013.1074

12. Steele E.J., Al-Muft S., Augustyn K.K. et al 2018. Cause of Cambrian Explosion: Terrestrial or Cosmic? Prog. Biophys. Mol. Biol. 136: 3-23, https://doi.org/10.1016/j.pbiomolbio.2018.03.004

13. Steele E.J., Gorczynski R.M., Lindley R.A., et al, 2019. Lamarck and Panspermia - On the Efficient Spread of Living Systems Throughout the Cosmos. Prog. Biophys. Mol. Biol. Aug 22 In Press. https://doi.org/ 10.1016/j.pbiomolbio.2019.08.010

14. Valley, J.W., Ushikubo, T., Reinhard, D.A., et al, 2014. Hadean age for a post-magma-ocean zircon confirmed by atom-probe tomography, Nature Geoscience, 7, 219-223 
15. Wainwright, M. et al (2003). Microorganisms cultured from the stratospheric air samples obtained at $41 \mathrm{~km}$, FEMS Microbiol Lett, 218, 161. 7.

16. Wainwright, M., Rose, C.E., Baker, A. J. et al (2015). Biological entities isolated from the two stratospheric launches-continued evidence for a space origin, Journal of Astrobiology and outreach, 3:2.

17. Wickramasinghe, N.C. and Rycroft, M.J., 2018, On the Difficulty of the Transport of Electrically Charged Submicron Dust from the Earth's Surface to the High Ionosphere Advances in Astrophysics, Vol. 3, No. 3, August 2018 https://dx.doi.org/10.22606/adap.2018.33003 